\title{
Effet de la Consommation de l'Amande de Cacao sur la Croissance et les Valeurs Moyennes des Métabolites Sériques Chez le Rat
}

\author{
Patricia Amenan Kouadio, \\ Laboratoire Nutrition et Pharmacologie, UFR Biosciences, \\ Université Félix Houphouët-Boigny, Abidjan, Côte d'Ivoire \\ Barthélemy Koffi Attioua,
}

Laboratoire de Chimie Organique et des Substances Naturelles, UFR-SSMT,

Université Félix Houphouët-Boigny, Abidjan, Côte d'Ivoire

Rosemonde Affouët Yao,

Marcel N'dri Kasse,

Hermann Kouame Yeboue,

Alexandre Zoho Bi Foua,

Ernest Kouakou Amoikon,

Laboratoire Nutrition et Pharmacologie, UFR Biosciences,

Université Félix Houphouët-Boigny, Abidjan, Côte d'Ivoire

Résumé

Cette étude a pour objectif d'évaluer l'effet de la consommation des amandes de cacao sur les caractéristiques nutritionnelles et sur la valeur moyenne des paramètres biochimiques sériques chez les rats en croissance. Pour ce faire, vingt et un rats mâles en croissance repartis en trois lots ont été nourris durant 21 jours, avec trois régimes alimentaires. Ainsi, un régime témoin à base de poudre de soja $(\mathrm{SO})$, un régime à base de poudre de cacao fermenté (PCF) et un régime à base de poudre de cacao de marque "Tafissa" (PCC), acheté dans le commerce, ont été mis en expérimentation. Le taux de protéines de ces régimes est fixé à $10 \%$. A la fin de l'expérience, les résultats révèlent que les rats nourris avec les régimes alimentaires à base de poudre d'amande de cacao PCF et PCC ont subi une perte de poids corporel du début jusqu'à la fin de l'expérimentation, tandis que ceux nourris avec le régime témoin (SO) ont une croissance normale. Cette étude montre qu'il n'y a pas de différence significative $(\mathrm{p}>0,05)$ entre les valeurs de triglycéride, de cholestérol total et de cholestérol HDL des rats soumis aux régimes PCF et PCC par rapport au témoin. Les taux sériques de cholestérol LDL des rats des régimes $\mathrm{PCF}$ et $\mathrm{PCC}$ sont significativement inférieurs $(\mathrm{p} \leq 0,05)$ à ceux des 
témoins (SO). De même, les valeurs de bilirubines conjuguées et de bilirubines totales des rats soumis au régime PCC sont significativement inférieures ( $\mathrm{p}$ $\leq 0,05$ ) à celles des témoins. Ces résultats impliquent que l'amande de cacao pourrait être un bon complément alimentaire car, sa consommation régulière a des effets bénéfiques sur la santé par son action contre la surcharge pondérale et les maladies cardiovasculaires.

Mots-clés : Amande De Cacao, Rats, Nutrition, Sang

\section{Effect of Consumption of Cocoa Almond on Growth and the Average Values of Serum Metabolites in the Rat}

\section{Patricia Amenan Kouadio,}

Laboratoire Nutrition et Pharmacologie, UFR Biosciences, Université Félix Houphouët-Boigny, Abidjan, Côte d'Ivoire

\section{Barthélemy Koffi Attioua,}

Laboratoire de Chimie Organique et des Substances Naturelles, UFR-SSMT,

Université Félix Houphouët-Boigny, Abidjan, Côte d'Ivoire

Rosemonde Affouët Yao, Marcel N'dri Kasse,

Hermann Kouame Yeboue, Alexandre Zoho Bi Foua, Ernest Kouakou Amoikon, Laboratoire Nutrition et Pharmacologie, UFR Biosciences, Université Félix Houphouët-Boigny, Abidjan, Côte d'Ivoire

\footnotetext{
Abstract

This study aims to evaluate the effect of cocoa almond consumption on the nutritional characteristics and on the average value of serum biochemical parameters in growing rats. To do this, twenty-one male rats growing in three lots were fed for 21 days, with three diets including a control diet based on soybean powder (SO), a diet based on fermented cocoa powder (PCF) and a commercial cocoa powder diet of brand "Tafissa" (PCC). The protein content of these diets is set at $10 \%$. At the end of the experiment, the results reveal that rats fed on cocoa almond powder diets PCF and PCC undergo body weight loss from the beginning to the end of the experiment,
} 
while that of those fed the control diet (SO) have normal growth. This study shows that there is no significant difference $(\mathrm{p}>0.05)$ between the triglyceride, total cholesterol and HDL cholesterol values of rats subjected to the PCF and PCC diets compared to the control. Serum LDL cholesterol levels in PCF and PCC rats were significantly $(\mathrm{p} \leq 0.05)$ lower than those of controls one (SO). The values of conjugated bilirubins and total bilirubins of the rats subjected to the PCC diet were significantly lower $(p \leq 0.05)$ than those of the controls. These results imply that cocoa almond could be a good dietary supplement because, its regular consumption has beneficial effects on health by its action against overweight and cardiovascular diseases.

Keywords: Cocoa Almond, Rats, Nutrition, Blood

\section{Introduction}

Selon la FAO (2004), 842 millions de personnes souffrent encore de la malnutrition dans le monde dont 798 millions dans les pays en voie de développement. La Côte d'Ivoire dispose d'atouts agronomiques considérables sur toute l'étendue du territoire, et sur toute l'année (PAM/FAO, 2009). Ses importantes potentialités naturelles lui ont permis d'exploiter une gamme de variétés de productions végétales parmi lesquelles, il y a le cacaotier, le caféier, l'anacardier, le manioc, l'igname, le bananier qui sont des cultures destinées au commerce extérieur. Cependant, malgré la disponibilité énorme des ressources alimentaires, l'accès aux aliments reste encore limité pour bon nombre de la population. La malnutrition demeure un obstacle pour le développement du pays (Ouedraogo, 2008). La Côte d'Ivoire produit $40 \%$ du cacao mondial (1500000 tonnes) dont la grande partie est destinée à l'exportation. Aussi, les produits finis du cacao tels que le chocolat ou la poudre de chocolat demeurent des aliments de luxe pour les populations ivoiriennes. L'amande de cacao suscite beaucoup d'intérêts auprès des chercheurs à cause de ses propriétés aux niveaux nutritionnelles et pharmacologiques (Dillinger et al., 2000 ; Lecumberri et al., 2007 ; Abril-Gil et al., 2012). Toutefois, ces travaux restent encore insuffisants pour une connaissance rationnelle des potentialités du cacao. Les recherches effectuées en Côte d'Ivoire sont plus axées sur la productivité et la vulgarisation du fruit. Très peu de travaux scientifiques ont porté sur l'évaluation des propriétés nutritionnelles de l'amande de cacao. Cette étude a été entreprise en vue de contribuer à l'évaluation du potentiel nutritionnel de l'amande de cacao, partie très convoitée de la fève. 


\section{Matériel et méthodes}

\subsection{Matériel végétal}

Le matériel végétal utilisé dans cette étude est constitué de trois lots de poudres. Le premier lot provient d'amandes de fèves de cacaotier (Theobroma Cacao L), de variété Forastero, collectées à Assakro, dans la région d'Abengourou (Est de la Côte d'Ivoire) puis fermentées et séchées avant d'être broyées. Le second lot est de la poudre de cacao commercial et enfin le dernier et troisième lot est de la poudre de soja qui a été achetée dans le commerce à Abidjan.

\subsection{Matériel animal et leurs traitements}

Le matériel animal utilisé est constitué de 21 rats en croissance de souche Wistar, ayant un poids moyen compris entre 62 - 65 g et âgés de 50 et 60 jours. Ces rats sont issus du vivarium de l'Ecole Normale Supérieure (ENS) d'Abidjan (Côte d'Ivoire). Les régimes expérimentaux sont préparés selon la méthode décrite par Garcin et al. (1984), avec des modifications. Ces régimes sont iso-protéiques $(10 \%)$ et iso-énergétiques $(4225 \mathrm{kcal} / \mathrm{kg}$ matière sèche). Les animaux ont été soumis à un régime de référence à base de soja ( $\mathrm{SO})$, à un régime à base de poudre de cacao fermenté $(\mathrm{PCF})$, fabriqué au laboratoire de Nutrition et Pharmacologie et enfin, à un régime à base de poudre de cacao acheté dans le commerce de marque "Tafissa" (100\% cacao) dénommé PCC. Tous ces régimes sont enrichis d'un prémix de vitamines et de minéraux, avec ajout d'eau pour l'homogénéisation (Tableau I). La cuisson des régimes est effectuée sur une plaque chauffante. A la fin de la préparation, les différents régimes obtenus sont conservés au réfrigérateur à $4{ }^{\circ} \mathrm{C}$. Les aliments frais sont pesés avant d'être servis aux rats.

Tableau I : Régimes alimentaires des rats

\begin{tabular}{lccc}
\hline \multirow{2}{*}{ Ingrédients } & \multicolumn{3}{c}{ Régimes alimentaires } \\
\cline { 2 - 4 } & SO & PCF & PCC \\
\hline Poudre de soja & 270,27 & 0,00 & 0,00 \\
Poudre de cacao & 0,00 & 482,86 & 0,00 \\
Poudre de cacao Tafissa & 0,00 & 0,00 & 526,32 \\
Fécule de maïs (g) & 423,74 & 361,14 & 167,68 \\
Sucre (g) & 250,00 & 250,00 & 250,00 \\
Prémix & 1,00 & 1,00 & 1,00 \\
Agar-agar & 5,00 & 5,00 & 5,00 \\
Huile & 50,00 & 50,00 & 50,00 \\
Total (g) & 1000,00 & 1000,00 & 1000,00 \\
Eau (mL) & 1000,00 & 1000,00 & 1000,00 \\
Energie (Kcal/MS) & 4225,00 & 4225,00 & 4225,00 \\
\hline
\end{tabular}

SO: régime témoin à base de soja; PCF: régime à base de poudre de cacao fermenté; PCC: régime à base de cacao du commerce. Le taux de protéines dans les régimes est fixé à $10 \%$; la valeur énergétique de chaque régime est 4225Kcal/Kg. Source : Garcin et al. (1984). 


\subsection{Méthode d'alimentation des rats}

Les paramètres de croissance sont présentés dans le Tableau II. Les animaux ont été répartis en trois groupes de sept rats, logés individuellement dans les cages à métabolisme individuelles. Ils ont été soumis chacun aux régimes alimentaires. Les régimes sont distribués ad libitum chaque jour, en vue de déterminer la quantité d'aliment ingéré. L'eau des biberons est aussi renouvelée. Les animaux sont pesés tous les deux jours afin de déterminer le gain de poids corporel. L'expérience a duré 21 jours.

A la fin de l'expérimentation, les rats sont soumis à un jeûne de 16 heures. Le lendemain, ils sont anesthésiés au chloral à $10 \%$, à raison de 3 $\mathrm{ml} / \mathrm{kg}$, puis sacrifiés. Environ $5 \mathrm{ml}$ de sang sont prélevés dans des tubes secs. Ces échantillons de sang sont centrifugés à 3000 tours, pendant 10 min, dans une centrifugeuse réfrigérée. Le sérum est prélevé et servira pour les dosages des métabolites sériques au laboratoire de Biochimie médicale du Centre Hospitalier Universitaire de Treichville (CHU-T), Abidjan (Côte d'Ivoire).

Tableau II : Expression des paramètres nutritionnels

\begin{tabular}{lr}
\hline Paramètres nutritionnels & $\begin{array}{c}\text { Expressions } \\
\text { mathématiques }\end{array}$ \\
\hline Matière sèche ingérée (MSI) & MSI $(\mathrm{g})=\frac{\mathrm{AI} \times \mathrm{MS}}{21}$ \\
$\begin{array}{l}\text { Gain de poids corporel } \\
(\mathrm{GPC})\end{array}$ & $\mathrm{GPC}(\mathrm{g})=\frac{\mathrm{PF}-\mathrm{PI}}{21}$ \\
$\begin{array}{l}\text { Coefficient d'efficacité } \\
\text { alimentaire (CEA) }\end{array}$ & $\mathrm{CEA}=\frac{\mathrm{GPC}(\mathrm{g})}{\mathrm{MSI}(\mathrm{g})}$ \\
$\begin{array}{l}\text { Protéine totale ingérée (PTI) } \\
\text { Coefficient d'efficacité } \\
\text { protéique (CEP) }\end{array}$ & $\mathrm{PTI}(\mathrm{g})=\mathrm{MSI} \times \mathrm{P} \%$ \\
\hline
\end{tabular}

21 : durée de l'expérience en jours ; MS : matière sèche ; $\mathbf{A I}$ : Aliment ingéré ;

$\mathbf{P I}$ : poids initial ; PF : poids final ; \% $\mathbf{P}$ : Pourcentage de protéines des régimes.

\subsection{Analyse et traitement des données}

Le programme informatique graphPad prism version 5 (San Diego Ca, USA) est utilisé pour l'analyse statistique des résultats. Les valeurs moyennes des données sont toujours suivies de leurs écart-types. La comparaison des moyennes est basée sur l'analyse de variance (ANOVA) suivie du test de Newman-Keuls (au seuil de $5 \%$ ). Les moyennes sont suivies des lettres a, b, c, d, e, etc. en super script. Les moyennes portant des lettres différentes sur la même ligne sont statistiquement différentes. Dans le cas contraire, ces moyennes ne sont pas différentes. 


\section{Résultats}

\subsection{Effet de la consommation de la poudre d'amande de cacao sur la croissance des rats}

L'évolution de la croissance des rats est illustrée sur la Figure 1. Cette figure montre qu'il y a une perte de poids corporel des rats qui ont les régimes alimentaires à base de poudre d'amande de cacao (PCF et PCC). Par contre, ceux dont le régime alimentaire est à base de poudre de soja $(\mathrm{SO})$ ont une croissance normale.

Les valeurs moyennes des caractéristiques nutritionnelles des rats sont mentionnées dans le Tableau III. Le poids final des rats soumis aux régimes alimentaires à base de poudre de cacao de types PCF $(57,26 \pm 11,55 \mathrm{~g})$ et PCC $(54,70 \pm 4,76 \mathrm{~g})$ est significativement inférieur $(\mathrm{p} \leq 0,05)$ à celui des rats soumis au régime à base de poudre de soja, pris comme régime témoin, $\mathrm{SO}$ $(113,87 \pm 5,28 \mathrm{~g})$. Les valeurs de la matière sèche ingérée (MSI) des rats soumis aux régimes PCF et PCC sont respectivement de 8,14 $\pm 2,55 \mathrm{~g} / \mathrm{j}$ et de $7,66 \pm 1,55$ $\mathrm{g} / \mathrm{j}$. Ces valeurs ne sont pas significativement différentes $(\mathrm{p}>0,05)$ de celles des rats témoins $(8,86 \pm 1,26 \mathrm{~g} / \mathrm{j})$. Cependant, les valeurs des gains de poids corporel (GPC) des animaux soumis aux régimes PCF $(-0,14 \pm 0,79 \mathrm{~g} / \mathrm{j})$ et PCC $(-0,52 \pm 0,40 \mathrm{~g} / \mathrm{j})$ sont plus faibles que celles des rats témoins $(2,34 \pm 0,27 \mathrm{~g} / \mathrm{j})$. De même, les valeurs de coefficient d'efficacité alimentaire (CEA) et de coefficient d'efficacité protéique (CEP) des rats des régimes PCF et PCC sont significativement inférieures $(\mathrm{p} \leq 0,05)$ à celles de $\mathrm{SO}$ (témoin).

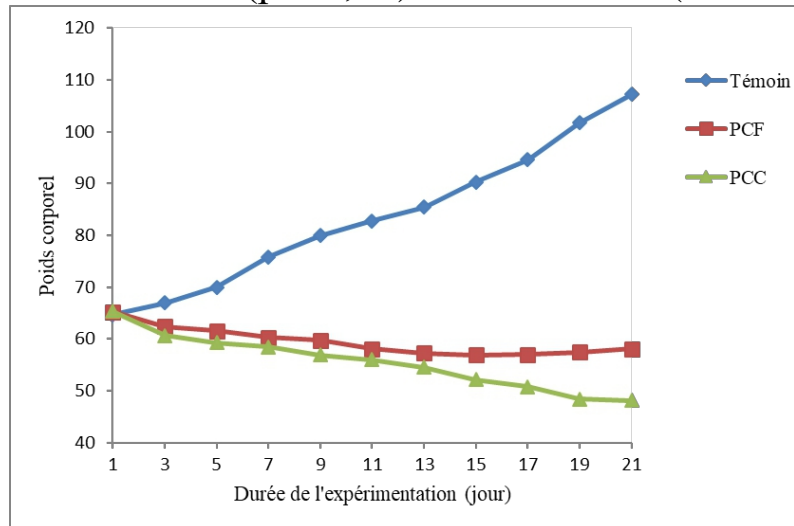

Figure 1 : Variation du poids corporel des rats en fonction des régimes alimentaires. $(\mathbf{n}=6)$ : Nombre de rats par traitement ; SO: Régime de référence soja ;

PCF: Régime alimentaire à base de poudre de cacao fermenté ;

PCC: Régime alimentaire à base de poudre de cacao du commerce. 
Tableau III : Valeur moyenne des caractéristiques nutritionnelles des rats consommant la poudre d'amande de cacao

\begin{tabular}{lccc}
\hline \multicolumn{1}{c}{ Critères } & \multicolumn{3}{c}{ Régimes alimentaires } \\
\cline { 2 - 4 } & Témoin & PCF & PCC \\
\hline Poids initial (g) & $64,66 \pm 6,75^{\mathrm{a}}$ & $65,19 \pm 6,16^{\mathrm{a}}$ & $65,33 \pm 5,75^{\mathrm{a}}$ \\
Poids final (g) & $113,87 \pm 5,28^{\mathrm{a}}$ & $57,26 \pm 11,55^{\mathrm{b}}$ & $54,70 \pm 4,76^{\mathrm{c}}$ \\
GPC (g/j) & $2,34 \pm 0,27^{\mathrm{b}}$ & $-0,14 \pm 0,79^{\mathrm{c}}$ & $-0,52 \pm 0,40^{\mathrm{d}}$ \\
MSI (g/j) & $8,86 \pm 1,26^{\mathrm{a}}$ & $8,14 \pm 2,55^{\mathrm{a}}$ & $7,66 \pm 1,55^{\mathrm{a}}$ \\
PTI (g/j) & $0,87 \pm 0,13^{\mathrm{a}}$ & $0,81 \pm 0,25^{\mathrm{a}}$ & $0,76 \pm 0,15^{\mathrm{a}}$ \\
CEA & $0,27 \pm 0,03^{\mathrm{b}}$ & $-0,02 \pm 0,01^{\mathrm{c}}$ & $-0,07 \pm 0,07^{\mathrm{d}}$ \\
CEP & $2,69 \pm 0,31^{\mathrm{a}}$ & $-0,18 \pm 1,07^{\mathrm{b}}$ & $-0,68 \pm 0,87^{\mathrm{c}}$ \\
\hline
\end{tabular}

L'analyse des variances est suivie du test de comparaisons multiples de NewmanKeuls au seuil de $5 \%$. Les moyennes sont suivies des lettres a, b, c, d, e,... en super

script ; les moyennes portant des lettres différentes sur la même ligne sont statistiquement différentes pour $\mathrm{P} \leq 0,05$. Témoin : Régime alimentaire à base de poudre de soja $; \mathbf{P C F}$ : Régime alimentaire à base de poudre d'amande de cacao fermenté ; PCC : Régime alimentaire à base de poudre de cacao commercial.

MSI : matière sèche ingérée ; PTI : protéines totales ingérées ;

GPC : Gain de poids corporel ; CEA : coefficient d'efficacité alimentaire ;

CEP : coefficient d'efficacité protéique.

\subsection{Effet de la consommation de la poudre de cacao sur la valeur moyenne des métabolites sériques chez les rats}

Le tableau IV donne les valeurs moyennes des métabolites sériques des rats. Les valeurs d'urée, d'acide urique, de créatinine et de glucose des rats soumis aux régimes alimentaires PCF et PCC ne sont pas significativement différentes $(p>0,05)$ de celles des témoins (SO). De même, les taux de cholestérol total, de cholestérol-HDL et de triglycérides des rats soumis aux régimes PCF et PCC ne sont pas différents de ceux des témoins. Cependant, le taux de cholestérol-LDL des rats soumis aux régimes PCF $(0,37 \pm 0,08 \mathrm{~g} / \mathrm{L})$ et PCC $(0,28 \pm 0,03 \mathrm{~g} / \mathrm{L})$ sont plus faibles $(\mathrm{p} \leq 0,05)$ que ceux des témoins $(0,51 \pm 0,06 \mathrm{~g} / \mathrm{L})$. Les valeurs de bilirubines conjuguées et de bilirubines totales des rats du régime PCC $(3,23 \pm 0,40 \mathrm{~g} / \mathrm{L} ; 12,33 \pm 1,58 \mathrm{~g} / \mathrm{L})$ sont plus faibles ( $\mathrm{p}$ $\leq 0,05)$ que celles des témoins $(4,87 \pm 0,83 \mathrm{~g} / \mathrm{L} ; 17 \pm 3 \mathrm{~g} / \mathrm{L})$. 
Tableau IV : Valeur moyenne des métabolites sériques des rats consommant la poudre de cacao

\begin{tabular}{lccc}
\hline Paramètres & \multicolumn{3}{c}{ Régimes alimentaires } \\
\cline { 2 - 4 } & $0,29 \pm 0,2^{\mathrm{a}}$ & $0,30 \pm 0,06^{\mathrm{a}}$ & $\mathbf{P C C}$ \\
\hline Urée (g/L) & $25,67 \pm 4,51^{\mathrm{a}}$ & $27,33 \pm 4,14^{\mathrm{a}}$ & $28 \pm 2,65^{\mathrm{a}}$ \\
Acide urique & $0,33 \pm 0,04^{\mathrm{a}}$ & $0,36 \pm 0,07^{\mathrm{a}}$ & $0,28 \pm 0,03^{\mathrm{a}}$ \\
Glucose (g/L) & $9,33 \pm 0,58^{\mathrm{a}}$ & $10,67 \pm 1,53^{\mathrm{a}}$ & $10,00 \pm 1,00^{\mathrm{a}}$ \\
Créatinine (mg/L) & $1,36 \pm 0,27^{\mathrm{a}}$ & $1,43 \pm 0,16^{\mathrm{a}}$ & $1,26 \pm 0,05^{\mathrm{a}}$ \\
Triglycérides (g/L) & $1,07 \pm 0,09^{\mathrm{a}}$ & $0,93 \pm 0,16^{\mathrm{a}}$ & $0,92 \pm 0,08^{\mathrm{a}}$ \\
Cholestérol-Total (g/L) & $0,27 \pm 0,05^{\mathrm{a}}$ & $0,28 \pm 0,03^{\mathrm{a}}$ \\
Cholestérol-HDL (g/L) & $0,29 \pm 0,02^{\mathrm{a}}$ & $0,37 \pm 0,08^{\mathrm{b}}$ & $0,38 \pm 0,06^{\mathrm{b}}$ \\
Cholestérol-LDL (g/L) & $0,51 \pm 0,06^{\mathrm{a}}$ & $14 \pm 2,65^{\mathrm{a}}$ & $12,33 \pm 1,58^{\mathrm{b}}$ \\
Bilirubines Totales & $17,00 \pm 3,00^{\mathrm{a}}$ & $3,70 \pm 0,52^{\mathrm{a}}$ & $3,23 \pm 0,40^{\mathrm{b}}$ \\
Bilirubines Conjuguées & $4,87 \pm 0,83^{\mathrm{a}}$ & $\mathbf{g}$ &
\end{tabular}

L'analyse des variances est suivie du test de comparaisons multiples de Newman-Keuls au seuil de $5 \%$. Les moyennes sont suivies des lettres a, b, c, d, e,... en super script ; les moyennes portant des lettres différentes sur la même ligne sont statistiquement différentes pour $\mathrm{P} \leq 0,05$. Témoin : Régime alimentaire à base de poudre de soja ;

PCF : Régime alimentaire à base de poudre de cacao fermenté ;

PCC : Régime alimentaire à base de poudre de cacao commercial.

\subsection{Effets de la consommation de la poudre de cacao sur les valeurs moyennes des électrolytes sériques chez les rats}

Le tableau $\mathrm{V}$ présente les effets de la consommation des régimes expérimentaux sur les valeurs des électrolytes sériques : $\mathrm{Ca}^{2+}, \mathrm{P}^{5+}, \mathrm{K}^{+}, \mathrm{Na}^{+}$et $\mathrm{Fe}^{3+}$. Les valeurs de $\mathrm{P}^{5+}$ et de $\mathrm{Fe}^{3+}$ des rats soumis aux régimes alimentaires à base de poudre de cacao PCF et PCC ne sont pas significativement différentes ( $p>0,05$ ) de celles des témoins ( $\mathrm{SO}$ ). Cependant, les valeurs de $\mathrm{Ca}^{2+}$ des rats nourris avec le régime PCC $(102,2 \pm 4 \mathrm{mEq} / \mathrm{L})$ sont significativement supérieures $(\mathrm{p} \leq 0,05)$ à celles des témoins $(94 \pm 2,36 \mathrm{mEq} / \mathrm{L})$. Celles de $\mathrm{Na}^{+}$ du régime PCF $(136,3 \pm 2,52 \mathrm{mEq} / \mathrm{L})$ sont significativement inférieures $(\mathrm{p} \leq$ $0,05)$ à celles des témoins $(141,70 \pm 3,05 \mathrm{mEq} / \mathrm{L})$. 
Tableau V : Valeurs moyennes des électrolytes sériques

\begin{tabular}{lccc|}
\hline Paramètres & \multicolumn{3}{c|}{ Régimes alimentaires } \\
\cline { 2 - 4 } & Témoin & PCF & PCC \\
\hline $\mathrm{Ca}^{2+}(\mathrm{mEq} / \mathrm{L})$ & $94,00 \pm 2,36^{\mathrm{a}}$ & $100,70 \pm 3,05^{\mathrm{a}}$ & $102,2 \pm 4,00^{\mathrm{b}}$ \\
$\mathrm{P}^{5+}(\mathrm{mEq} / \mathrm{L})$ & $0,33 \pm 0,04^{\mathrm{a}}$ & $0,36 \pm 0,07^{\mathrm{a}}$ & $0,28 \pm 0,03^{\mathrm{a}}$ \\
$\mathrm{K}^{+}(\mathrm{mEq} / \mathrm{L})$ & $9,33 \pm 0,58^{\mathrm{a}}$ & $10,67 \pm 1,53^{\mathrm{a}}$ & $10,00 \pm 1,00^{\mathrm{a}}$ \\
$\mathrm{Na}^{+}(\mathrm{mEq} / \mathrm{L})$ & $141,70 \pm 3,05^{\mathrm{a}}$ & $136,3 \pm 2,52^{\mathrm{b}}$ & $141,00 \pm 1,73^{\mathrm{a}}$ \\
$\mathrm{Fe}^{3+}(\mathrm{mEq} / \mathrm{L})$ & $6,73 \pm 1,88^{\mathrm{a}}$ & $6,66 \pm 0,98^{\mathrm{a}}$ & $7,32 \pm 0,99^{\mathrm{a}}$ \\
\hline
\end{tabular}

L'analyse des variances est suivie du test de comparaisons multiples de Newman-Keuls au seuil de $5 \%$. Les moyennes sont suivies des lettres a, b, c, d, e,... en super script ; les moyennes portant des lettres différentes sur la même ligne sont statistiquement différentes pour $\mathrm{P} \leq 0,05$. Témoin: Régime alimentaire à base de poudre de soja ;

PCF: Régime alimentaire à base de poudre d'amande de cacao fabriqué au laboratoire ;

PCC: Régime alimentaire à base de poudre de cacao commercial.

\section{Discussion}

Les résultats des paramètres nutritionnels montrent que les rats nourris avec les régimes alimentaires à base de poudre d'amande de cacao PCF et PCC subissent une perte de poids corporel du début jusqu'à la fin de l'expérimentation. Des résultats similaires ont été rapportés par Ramiro-Piug et al. (2008) et Olooto et al. (2015). Ces auteurs ont montré que l'ingestion de la poudre de cacao réduit le poids corporel des animaux durant le traitement.

L'appétence des rats soumis aux régimes alimentaires à base de poudre d'amande de cacao est bonne. En effet, la quantité de matière sèche ingérée par les rats des régimes PCF et PCC est comparable à celle des témoins. Ces résultats sont en accord avec ceux de Perez-Berezo et al. (2011) et MassotCladera et al. (2012a) ; qui ont montré que la perte de poids observée chez les rats consommant le cacao n'est donc pas liée au taux faible de matière sèche ingérée puisque leur consommation est comparable à celle des témoins. Cette même tendance est observée au niveau des protéines totales ingérées (PIT). Les valeurs des protéines totales ingérées (PIT) des rats soumis aux régimes alimentaires PCF et PCC ne sont pas significativement différentes ( $p>0,05)$ de celles des témoins (SO), alors que ces rats ont une faible croissance par rapport à celle des témoins. Selon Chater et al. (2015), la qualité des protéines ingérées dépend des acides aminés et de la digestibilité de ces protéines. De même, Ces régimes à base de poudre d'amande de cacao (PCF, PCC) provoquent des valeurs de coefficients d'efficacité alimentaire (CEA) et de coefficient d'efficacité protéique (CEP) très négatives. Massot-Cladera et al. (2017b) ont montré que la mauvaise performance des protéines est due à la présence de composés bioactifs dans le cacao qui pourraient influencer l'efficacité des protéines. Ces composés présents dans le cacao vont entraîner la diminution de la teneur en acide aminé disponible (Hurrell et Fiont, 1982). Les méthylxanthines telles que les théobromines contenues dans l'amande de 
cacao sont aussi responsables de l'amaigrissement des rats. La théobromine, à concentration élevée provoque une baisse du poids corporel des sujets. Elle altère la stimulation de la synthèse protéique des animaux (Massot-Cladera et al., 2017b). De plus, les tanins du cacao sont également responsables de cet amaigrissement. En effet, l'analyse chimique de l'amande de cacao montre que le cacao à une teneur élevée en tanins. Ces tanins présents dans l'amande de cacao sont capables de retarder la croissance des rats, par la réduction de la digestion et/ou l'absorption des acides aminés et des minéraux (Laurena et al., 1984).

Les valeurs moyennes des métabolites sériques des rats expérimentaux (PCF, PCC) ont été déterminées. Les valeurs d'urée et de créatinine des rats consommant les régimes PCF et PCC ne présentent aucune différence significative $(\mathrm{p}>0,05)$ de celles des témoins. Ces résultats sont comparables à celles trouvées par Dally et al. (2014) lors de ses travaux sur les mets ivoiriens, qui ont montré que les valeurs de la créatine et d'urée des rats soumis aux mets ivoiriens ne sont pas affectées par les facteurs extra-rénaux. Le taux de triglycérides, de cholestérol total et de cholestérol HDL des animaux nourris avec les régimes PCF et PCC n'ont pas subi de variation et ils sont identiques à celui des témoins. Les valeurs de tous ces paramètres lipidiques montrent bien la qualité nutritionnelle de l'amande de cacao. De plus, on observe une diminution de la concentration sérique de cholestérol LDL des rats nourris avec les régimes PCF et PCC comparativement à celle des rats témoins. Ces résultats sont similaires à ceux rapportés par Grassi et al. (2008) qui ont noté une diminution du taux de cholestérol LDL chez les sujets, après 15 jours de consommation du chocolat foncé.

Le taux sérique de sodium des rats nourris avec le régime témoin est comparable à celui des rats nourris avec le régime PCC. Cependant, ce taux est significativement différent de celui des rats nourris avec le régime PCF. Les taux sériques de potassium, de phosphore, de fer des rats consommant les différents régimes alimentaires PCF et PCC, ne sont pas différents ( $p>0,05)$ de ceux des témoins (SO). Ces mêmes régimes à base de cacao ont des taux sériques de $\mathrm{Ca}^{2+}$ inférieurs à celui des témoins.

\section{Conclusion}

$\mathrm{Au}$ terme de cette étude, il ressort que la consommation de la poudre d'amande de cacao (PCF et PCC) induit une réduction du poids corporel des rats. Par contre, la consommation des amandes de cacao n'entraîne pas de perturbation au niveau des valeurs de créatinine, d'urée, d'acide urique, de triglycérides, de cholestérol total, de cholestérol-HDL et de glucose. La consommation d'amande de cacao peut donc constituer une thérapie nutritionnelle efficace dans la prévention et les traitements des surcharges pondérales. 


\section{References:}

1. Abril-Gil M., Massot-Cladera M., Pérez-Cano F.J., Castellote C., Franch À. \& Castell M. (2012). A diet enriched with cocoa prevents IgE synthesis in a rat allergy model. Journal of Pharmacology Research, 65 (6): 603-608.

2. Chater P., Wilcox M.D., Pearson J.P. \& Brownlee I.A. (2015). The impact of dietary fibres on the physiological processes governing small intestinal digestive processes. Bioactive Carbohydrates and Dietary Fibre, 6 (2): 117-132.

3. Dally T., Méité A., Ouattara H., Kouamé G.K. \& Kati-Coulibaly S. (2014). Nutritionnal quality of three Ivoirians foods consumed: Biochimical seric studies on growing rats (Wistar). Parkistan Journal of Nutrition, 13 (5): 271-274.

4. Dillinger T.L., Barriga P., Escarcega S., Jimenez M., Lowe D.S. \& Grivetti L. E. (2000). Food of the Gods: Cure for humanity? A cultural history of the medicinal and ritual use of chocolate. Journal of Nutrition, 130 (8) : 2057-2072.

5. FAO (2004). La situation mondiale de l'alimentation et de l'agriculture. Collection Fao N³5 Rome : 244-264.

6. Garcin H., Higueret P. et Amoikon K. (1984) "Effect of large dose of retinol or retinoid acid on thyroid hormones in the rats". Annals of Nutrition and Metabolism, $28: 92-100$.

7. Grassi D., Desideri G., Necozione S., Lippi C., Casale R., Properzi G., Blumberg J.B. \& Ferri C. (2008). Blood pressure is reduced and insulin sensitivity increased in glucose intolerant, hypertensive subjects after 15 days of consuming high-polyphenol dark chocolate. Journal of Nutrition, 138: 1671-1676.

8. Hurrell R.F. \& Fiont P.A. (1982). Protein-polyphenol reactions. 1. Nutritional and metabolic consequence of the reaction between oxidized caffeic acid and the lysine residues of casein. British Journal of Nutrition, 47: 191-211.

9. Laurena A.C., Van Den T. \& Mendoza E.M.T. (1984). Effects of condensed tannins on in vitro digestibility cowpea (Vigna unguiculata (L.). Journal Agricultural and Food Chemistry, 32: 1045-1048.

10. Lecumberri E., Mateos R. \& Izquierdo-Pulido M. (2007). Dietary fibre composition, antioxidant capacity and physico-chemical properties of a fibre-rich product from cocoa (Theobroma cacao L.). Food Chemistry, 104: 948-954.

11. Massot-Cladera M., Franch À., Castell M., Francisco J., Pérez Cano. (2017b). Cocoa polyphenols and fiber modify colonic gene expression in rats. European Journal Nutrition, 56: 1871-1885. 
12. Massot-Cladera M., Pérez-Berezo T., Franch À., Castell M. and PérezCano F. J. (2012a). Cocoa modulatory effect on rat faecal microbiota and colonic cross talk. Archive of Biochemistry and Biophysics, 527:105-112

13. Olooto E.W., Amballi A.A., Onakomaya A.O., Olawale O.O. \& Ojo O.T. (2015). Effect of the aqueous cocoa powder extract on the biochemical and haematological of female albinos rat. Annals Biological Sciences, 2: 10-18

14. Ouedraogo E. (2008). Le bilan alimentaire par les produits végétaux en Côte d'Ivoire est positif.132p.

15. PAM/FAO, (2009). Côte d'Ivoire. Etudes de l'impact de la hausse des prix sur la situation alimentaire des ménages-Région Centre-NordOuest et sud.23 au 28 Mars.

16. Perez-Berezo T., Franch A., Ramos-Romero S., Castellote C., PerezCano F. J. \& Castell M. (2011). "Cocoa-enriched diets modulate intestinal and systemic humoral immune response in young adult rats," Molecular Nutrition and Food Research, 55 (1) 56-66.

17. Ramiro-Puig E., Pérez-Cano F.J., Ramos-Romero S., Pérez-Berezo T., Castellote C., Permanyer J., Franch A., Izquierdo-Pulido M. \& Castell M. (2008). Intestinal immune system of young rats influenced by cocoa-enriched diet. Journal of Nutrition Biochemical ,19: 555-565. 